\title{
Numerical study of the effect of structure and geometry on van der Waals forces
}

\author{
C E Román-Velázquez and Bo E Sernelius \\ Dept. of Physics, Chemistry, and Biology, Linköping University, SE-581 83 \\ Linköping, Sweden \\ E-mail: roman@ifm.liu.se
}

\begin{abstract}
We use multipolar expansions to find the force on a gold coated sphere above a gold substrate; we study both an empty gold shell and a gold coated polystyrene sphere. We find four characteristic separation ranges. In the first region, which for the empty gold shell occurs for distances, $d$, smaller than the thickness of the coating, the result agrees with that on a solid gold sphere and varies as $d^{-2}$; for larger separations there is a region where the force behaves as if the coating is strictly two dimensional and varies as $d^{-5 / 2}$; in the third region the dependence is more unspecific; in the forth region when $d$ is larger than the radius, the force varies as $d^{-4}$. For homogeneous objects of more general shapes we introduce a numerical method based on the solution of an integral equation for the electric field over a system of objects with arbitrary shapes. We study the effect of shape and orientation on the van der Waals interaction between an object and a substrate and between two objects.
\end{abstract}

\section{Introduction}

In his seminar paper [1] Casimir calculated the energy between two parallel perfect conductor half spaces as the change of zero-point energy of the classical electromagnetic field,

$$
\mathcal{U}(z)=\frac{\hbar}{2} \sum_{i}\left[\omega_{i}(z)-\omega_{i}(z \rightarrow \infty)\right]
$$

where $\omega_{i}(z)$ are the frequencies of the electromagnetic field modes of the plates separated by a distance $z$. This procedure has been employed by van Kampen [2] to study vdW (van der Waals) forces of dielectric plates in the non-retarded case. Later Gerlach [3] interpreted the retarded Casimir forces in terms of interacting surface plasmons of the plates. Most recently the problem of Casimir energy between two dielectric plates has been revisited and it has been shown that it can be obtained as the sum of contributions from the interacting surface plasmons and from the propagating modes of a cavity formed by the parallel plates $4,4,6,6$. Apart from the original results for parallel plates and slabs, a limited number of results exists for other geometries and combination of materials. The most well known result is the so called proximity force approximation (PFA) 7]. In that model the interaction between objects of various 
shapes is calculated in terms of the energy between planar interfaces. In the case of a spherical object of radius $R$, above a planar substrate, the force $F$ becomes

$$
F(z)=-\frac{d V(z)}{d z}=2 \pi R E_{p}(z)\left[1-\frac{1}{R E_{p}(z)} \int_{z}^{z+R} d h E_{p}(h)\right],
$$

where $z$ is the closest distance and $E_{p}(h)$ is the interaction energy per unit area between two planar interfaces a distance $h$ apart. The first factor of the result is what one usually means with the PFA. The second factor is a correction which we neglect in this work. In the case of $\mathrm{vdW}$ forces other analytical results exist for highly symmetric geometries. One of them is the following general expression for the interaction energy between two cylinders, spheres or half spaces at small separations

$$
E_{\mathrm{vdW}}=-A_{12}(\omega)\left[\frac{2 \pi R_{1} R_{2}}{R_{1}+R_{2}}\right]^{1-n / 2} \Gamma(1+n / 2) L^{n} d^{-(1+n / 2)},
$$

where $n=0$ for spheres, $n=1$ for cylinders, $n=2$ for half spaces and $A_{12}$ is the so called Hamaker constant given by

$$
A_{12}(\omega)=\frac{\hbar}{32 \pi^{2}} \sum_{l=1}^{\infty} \frac{1}{l^{3}} \int_{-\infty}^{\infty} d \omega\left[\frac{\varepsilon_{1}(\omega)-\varepsilon_{a}(\omega)}{\varepsilon_{1}(\omega)+\varepsilon_{a}(\omega)} \frac{\varepsilon_{2}(\omega)-\varepsilon_{a}(\omega)}{\varepsilon_{2}(\omega)+\varepsilon_{a}(\omega)}\right]^{l} .
$$

It has been shown that for larger separations, contrary to this formula, the energy is not symmetric with the interchange of materials between the objects 8 . The results of (3) in fact coincide with those obtained with the usual PFA approach for those geometries, in the nonretarded case which we are concerned with here.

Another set of results use additivity of pair interactions between the atoms of two objets to obtain the behavior of the force at close and at long distances. In spite of the well known fact that the Casimir force is not an additive interaction, there is a general consensus that these results give the correct distance dependence of the forces. One example is that for two cylinders; for parallel cylinders the vdW energy goes like $\sim d^{-1.5}$ when $d \rightarrow 0$, but when they are crossed, a dependence $\sim d^{-2}$ is expected, which is the same as between two spheres.

A spectral representation formalism has been employed [9] to obtain the resonance frequencies of a sphere above a substrate from which one obtains the vdW energy. In the spectral representation formalism, the optical response of a two face system is expressed as the sum of resonant terms from which it is possible to explicitly obtain its resonance frequencies [10. Those resonances correspond to the surface plasmons of the system. Furthermore, in that formalism the effects of geometry and material are clearly separated; this makes it possible to deduce the importance of the geometry in the resonances of the system, and consequently in its vdW energy.

In this work we use a multipolar method similar to that in [9] to calculate the $\mathrm{vdW}$ interaction between a substrate at arbitrary separation from a spherical object with an internal structure in the form of a sphere with a coat of a different material. We must mention that there are no results for vdW interactions between finite objects with a structure formed by parts of different materials. We also introduce a formalism for the determination of the vdW energy of a system of objects of arbitrary shape, separation and orientation. We present results for the vdW forces between cubes, and finite cylinders above substrates, and develop calculations of lateral and rotational forces between objects. 
The effect of structure and geometry on vdW forces

\section{Formalism}

\subsection{Multipolar method}

First we consider the system shown in the inset of figure 1(a). A system of four materials bounded by two concentric spheres of radii $R-\delta$ and $R$ with the center of the spheres at a distance $R+z$ from the surface of a substrate. The material of the interior of the sphere with radius $R-\delta$ has a dielectric function $\varepsilon_{i}(\omega)$; the coat or shell bounded by $R-\delta$ and $R$ contains a material with dielectric function $\varepsilon_{c}(\omega)$; the substrate has a dielectric function $\varepsilon_{s}(\omega)$. The full sphere is immersed in an ambient with dielectric function $\varepsilon_{a}(\omega)$. It has been shown [9, 11] that the multipolar moment $Q_{l m}$ of the surface charge induced by the fluctuations of the electromagnetic field with multipolar component $V_{l m}^{v a c}$ of the stochastic vacuum field, satisfies the system of linear equations

$$
\sum_{l^{\prime}}\left[\frac{1}{\alpha_{l}} \delta_{l, l^{\prime}}+f_{c} A_{l, l^{\prime}}^{m}(z)\right] Q_{l^{\prime} m}=-V_{l m}^{v a c}, \quad|m| \leq l, l^{\prime},
$$

where $\alpha_{l}$ is the polarizability of the sphere (independent of $m$ ), $f_{c}=$ $\left[\epsilon_{s}(\omega)-\epsilon_{a}(\omega)\right] /\left[\epsilon_{s}(\omega)+\epsilon_{a}(\omega)\right]$, and $A_{l, l^{\prime}}^{m}$ are the terms, of the matrix that relate the multipolar functions between the different systems of spherical coordinates of the sphere and its image, that vanish when $z \rightarrow 0$ [11, 9. For a homogeneous sphere $\alpha_{l}$ is given by

$$
\alpha_{l}^{m}=-\frac{c_{l}}{u(\omega)-n_{l}}, \quad c_{l}=l a^{l+1 / 2} /(2 l+1), \quad n_{l+1}=l /(2 l+1),
$$

where $a$ is the radius of the sphere and $u(\omega)=\left[1-\varepsilon_{s p}(\omega) / \varepsilon_{a}(\omega)\right]^{-1}$ is the so called spectral variable. The function $\varepsilon_{s p}(\omega)$ is the dielectric function of the sphere material. From (5) and (6) one can obtain a spectral representation for $Q_{l m}$,

$$
Q_{l m}=\sum_{l^{\prime}}\left[\sum_{s} \frac{C_{s ; l, l^{\prime}}^{m}(z)}{u(\omega)-n_{s}^{m}(z)}\right] V_{l^{\prime}, m}^{v a c}
$$

The strengths $C_{s ; l, l^{\prime}}^{m}=\sqrt{c_{l} c_{l^{\prime}}} U_{s, l}^{m} U_{s, l^{\prime}}^{m}$ are the so called spectral functions, where $U_{s, l}^{m}$ is the orthogonal matrix that satisfies

$$
\sum_{l, l^{\prime}} U_{s, l}^{m} H_{l, l^{\prime}}^{m}(z) U_{s^{\prime}, l^{\prime}}^{m}=n_{s}^{m}(z) \delta_{s, s^{\prime}} .
$$

Here, $H_{l, l^{\prime}}^{m}(z)=n_{l}^{0} \delta_{l l^{\prime}}+f_{c} \sqrt{c_{l}} \sqrt{c_{l^{\prime}}} A_{l, l^{\prime}}^{m}(z)$ is a real symmetric matrix. The resonance frequencies are given by $u\left(\omega_{s}^{m}\right)=n_{s}^{m}$, and the vdW energy is obtained from (5). Note that the matrix $H_{l, l^{\prime}}^{m}$ (and consequently $n_{s}$ ) depends only on the geometrical properties of the system and the dielectric properties of the substrate. In the case of a coated sphere the polarizability can be obtained using the boundary conditions of the electric potential as

$\alpha_{l}=-n_{l} R^{2 l+1} \frac{\left[n_{l}-u_{i c}(\omega)\right]-\left[n_{l}-u_{a c}(\omega)\right](1-\delta / R)^{2 l+1}}{\left[n_{l}-u_{i c}(\omega)\right]\left[n_{l}-u_{c a}(\omega)\right]+n_{l}\left(1-n_{l}\right)(1-\delta / R)^{2 l+1}}$.

with $u_{x y}(\omega)=1 /\left[1-\varepsilon_{x}(\omega) / \varepsilon_{y}(\omega)\right]$. Now it is neither possible to find a spectral representation of the system nor to obtain its resonances explicitly. One must rely on the argument principle to calculate the energy of the system, as follows. The determinant of (5D) becomes zero at the resonance frequencies of the system,

$$
G\left(\omega_{s}, z\right)=\operatorname{det}\left[\frac{1}{\alpha_{l}\left(\omega_{s}\right)} \delta_{l, l^{\prime}}+f_{c} A_{l, l^{\prime}}^{m}(z)\right]=0 .
$$


It is possible to apply the argument theorem to the multipolar problem [8] and using the fact that when $z \rightarrow \infty$ the resonances are those of the isolated coated sphere to find that (10) is given by

$$
\mathcal{U}(z)=-\frac{\hbar}{i 4 \pi} \int_{-i \infty}^{i \infty} d \omega \log \left[G(\omega, z) / \prod_{l} \frac{1}{\alpha_{l}(\omega)}\right] .
$$

This expression can also be used in the case of experimental dielectric functions or complex valued model dielectric functions for the sphere and substrate.

\subsection{Integral equation method}

The multipolar method above gives very high precision results over a wide range of distances. However to deal with particles of other shapes the multipolar method becomes much more complex and expensive numerically. On the other hand for finite objects of arbitrary geometry it is not possible to define one unique way of calculating the energy in the PFA approach. For spheres and cylinders one considers small planes that interact with the opposite in the direction parallel to the line that joins the centers of the objects. However for more complex geometries it is not clear how to define the interacting planes. R Fuchs has developed a spectral representation formalism for the calculation of the optical response, in the non-retarded limit, of homogeneous objects of arbitrary shapes [12. We use his basic formalism for the formulation of a model to obtain the vdW interaction energy for systems of objects of arbitrary geometry.

Fuchs has shown that the surface charge density, $\sigma$, satisfies the integral equation defined over the surface $\Sigma$ of the object [12],

$$
\mathbf{E}^{v a c}(\mathbf{r}, \omega) \cdot \hat{\mathbf{n}}(\mathbf{r})=2 \pi \frac{\varepsilon(\omega)+1}{\varepsilon(\omega)-1} \sigma(\mathbf{r})-\int_{\Sigma} \frac{\hat{\mathbf{n}}(\mathbf{r}) \cdot\left(\mathbf{r}-\mathbf{r}^{\prime}\right)}{\left|\mathbf{r}-\mathbf{r}^{\prime}\right|^{3}} \sigma(\mathbf{r}),
$$

where $\mathbf{E}^{v a c}$ is the stochastic vacuum field that excites the particle and $\hat{\mathbf{n}}$ is the surface normal pointing outwards from the surface. The complete deduction of (12) is independent of the form and connectivity of the surface [12. We use this integral equation when $\Sigma$ is the combination of the surfaces of two finite arbitrary objects. The simplest numerical solution of (12) is obtained, as in [12, by dividing the surface $\Sigma$ in small fragments of area $\Delta s_{i}$ and surface normal $\hat{\mathbf{n}}_{i}$ and with homogeneous surface charge $\sigma_{i}$, centered at the points $\mathbf{r}_{i}$. As a result, one obtains the linear system of equations,

$2 \pi \frac{\varepsilon(\omega)+1}{\varepsilon(\omega)-1} \sigma_{i}-\sum_{j} R_{i, j} \sigma_{i}=f_{i}, \quad R_{i, j}=\left(1-\delta_{i, j}\right) \frac{\hat{\mathbf{n}}_{i} \cdot\left(\mathbf{r}_{i}-\mathbf{r}_{j}\right) \Delta s_{i}}{\left|\mathbf{r}_{i}-\mathbf{r}_{j}\right|^{3}}$,

and $f_{i}=\mathbf{E}^{0}\left(\mathbf{r}_{i}\right) \cdot \hat{\mathbf{n}}\left(\mathbf{r}_{i}\right)$. A spectral representation can be obtained from solving (13),

$$
\sigma_{i}=-\sum_{j, s} \frac{C_{s}^{i, j}}{u(\omega)-n_{s}} f_{j}
$$

where $n_{s}=\left(1-m_{s} / 2 \pi\right) / 2, m_{s}$ are the proper values of the matrix $R_{i, j}$, and $C_{s}^{i, j}$ are obtained from the matrix that diagonalizes $R_{i, j}[12$. The resonance frequencies are given by $u\left(\omega_{s}\right)=n_{s}$ and like in the case of the multipolar spectral representation of a sphere above a substrate, the vdW energy could be calculated with (5). 


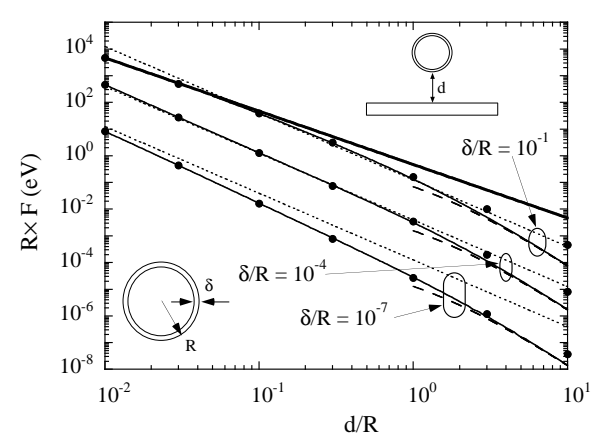

(a)

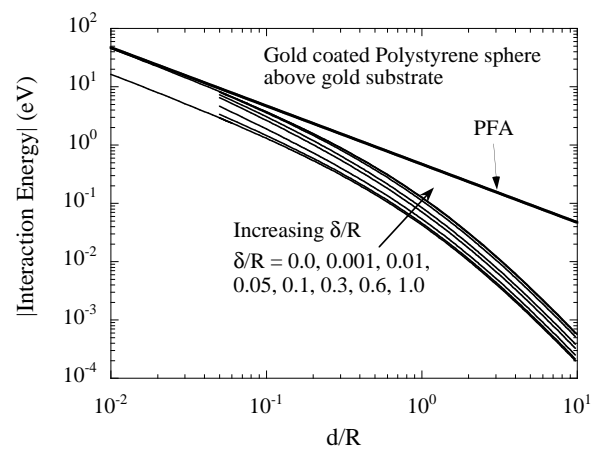

(b)

Figure 1. (a) The radius times the force on a gold shell of radius $R$ and thickness $\delta$ at distance $d$ above a gold substrate as function of $d / R$. See the text for details. (b) The interaction energy for a gold coated polystyrene sphere of radius $R$ and coat thickness $\delta$ at distance $d$ above a gold substrate as function of $d / R$. The coat thickness takes on the values $0.0,0.001,0.01,0.05,0.1,0.3,0.6$ and 1 , in units of $R$, counted from below. The thick solid curve is the PFA result based on two gold half spaces.

\section{Results and Discussion}

\subsection{Free standing spherical shells and gold coated polystyrene spheres}

For the calculations in this part we first construct the matrix of the linear system, (5), for a given $z / R$. For the dielectric function of gold and polystyrene we use those in 13 and [14, respectively. We calculate numerically the determinant and perform the integration of (11). An increasing maximum number $L_{\max }\left(l, l^{\prime}<L_{\max }\right)$ of multipoles is considered until the convergency of the energy is reached.

In figure 1(a) we show the results for a free standing gold shell above a solid gold substrate. As long as one may neglect retardation effects the figure is universal, independent of the size of the sphere. The thick solid curve is the PFA result when $E_{p}(z)$, of (2), is the interaction energy between two half spaces; here the force varies as $d^{-2}$. We see from the figure that the full result from using the multipolar method, thin solid curves, merge with the thick solid curve for small enough separations. This only happens inside the figure for the thickest of the coatings. For the other two examples it happens for smaller separations. For larger separations there is a region where the result follows the PFA result when $E_{p}(z)$ is the interaction energy between one half space and one strictly two dimensional film, dotted curves; here the force varies as $d^{-5 / 2}$, i.e., has a fractional power dependence. The dotted curves are obtained as [15, 16 ]

$$
R \times F=2 \pi R^{2} E_{p}(d) \approx 0.1556 \sqrt{n \hbar^{2} e^{2} / m_{e}} \sqrt{\delta / R} /(d / R)^{5 / 2} .
$$

The circles are the PFA result when $E_{p}(z)$ is the interaction energy between a half space and a film of thickness $\delta$. Note that the last two calculations (dotted lines and circles) are extensions of the PFA model; in the original model one used $E_{p}(z)$ of two half spaces. The dashed curves are the result from only including dipolar interactions.

Measurements of the vdW and Casimir interactions often involve spheres since in this case the difficult problem of alignment is obsolete. In most cases these spheres 


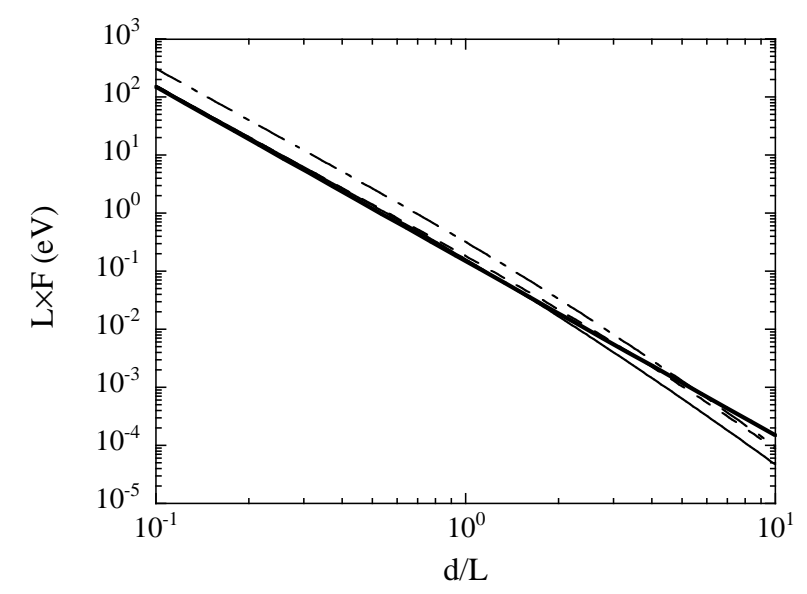

Figure 2. vdW forces between gold prisms the distance $d$ above a gold substrate. The thin solid curve is the result for a gold cube of side $L$. The dashed (dashdotted) curve is the result for a standing up (lying down) prism of length $2 L$. The thick solid curve is the PFA result for the cube, or standing up prism, based on two gold half spaces.

are not solid but coated; one assumes that the coated spheres may represent solid spheres. As we have seen, this assumption is only valid for relatively small distances. In figure 1(b) we show the interaction energy for a gold coated polystyrene sphere above a gold substrate. This way of displaying the results also provides universal curves, independent of the radius of the sphere. The radius, $R$, is the value for the full sphere including the coating. We see that only the spheres with very thick coatings behave as solid gold spheres.

\subsection{Interaction between objects of arbitrary geometry}

3.2.1. Objects above substrates: For the calculation of the vdW energy of these systems we define a discretization of the surface of the object in small areas specified by their positions, normal vectors and sizes. With that information we construct the matrix $R_{i, j}$ in (13) and obtain the eigenvalues $m_{s}$ numerically and from them the resonance frequencies. Finally, the energy is calculated with (5). Using a maximum of 1000 discrete elements per object we reached down to distances of $2 \%$ of its maximum diameter, within a numerical error of less than $2 \%$, as estimated from comparisons with calculations of $[8,9$.

In figure 2 we show calculations for different prisms above substrates, of the same material. The thin solid line shows the result for a cube of side $L$ at a distance $d$; the dashed line shows the result for a prism of height $2 L$ and square base with side length $L$; the thick solid line shows the result of (3). We note that when the cube and the square prism are very close to the substrate the force equals the base area times the force per unit area between two half spaces. At long distance the force is dominated by dipolar interactions which are proportional to the volume. The dash-dotted line shows the result when the prism is lying down, i.e., one of the rectangular sides is parallel to the substrate. So for small separations the force on the cube and standing up prism are equal while it is twice the size on the lying down prism; for large separations the forces on the standing up and lying down prisms are equal but the force on the cube 


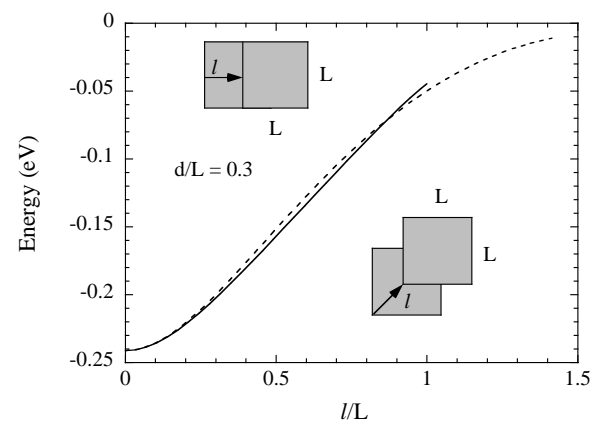

Figure 3. The lateral energy for two gold square-based prisms.

is only half the size. The result for a cylinder with height $L$ and a circular base with radius $R=L / \sqrt{\pi}$ follows closely the result for the cube, with a deviation smaller than the numerical error $\sim 1 \%$. These results show that the shape of the side that faces the substrate is not important. One consequence of this result is the additivity of the vdW energy for prisms: At all distances the vdW energy for prisms of same height and arbitrary shape is approximately proportional to the area of its base.

3.2.2. Interactions between two objects: The dependence of the $\mathrm{vdW}$ interaction on the object shapes becomes more complex in the case of the interaction between two objects. The interaction tends to modify the position and orientation of the particles to minimize the energy. It is possible to consider configurations in which two important kinds of interaction arise: lateral forces and rotational forces.

In figure 3 we show results for lateral forces between two prisms of height $L / 2$ with square bases of side $L$; they are placed above each other at a fixed distance $d$; they are displaced in the horizontal plane a distance $l$ from the complete parallel alignment. The solid line shows the results when the prisms are displaced in the direction parallel to one of its side faces. The dashed line shows the result when the prisms are displaced in the diagonal direction relative its base (see the lower inset of figure 3.) We note that both curves reach a minimum in the position of complete alignment. From this one can expect an oscillatory behaviour around this position. Even thought one could expect the additivity of the interaction in the limit when the particles are close to each other, there is no such additivity with the conditions considered here. In the diagonal displacement the area of interaction increases differently from that in the other type of displacement. However the energy shows an increase similar to that for the other displacement. This result suggests that the surface and boundary effects due to the interaction between the surface plasmons of the different objects is more important in the generation of lateral forces than in the case of an object above a substrate.

In figure 4 we show results for the rotational energy between two crossed cylinders as shown in the inset. The cylinders are rotated relative each other an angle $\theta$ around an axis passing through their centers. The angle varies from 0 to $\pi / 2$. The upper two curves are for cylinders with circular cross section with diameter $L$. The dotted (solid) curve is for cylinders of length $L(2 L)$. The lower two curves are for cylinders with square cross section of side length $L$. The dashed (dash-dotted) curve is for cylinders of length $L(2 L)$. In all cases the closest distance, $d$, is $0.3 L$.

We see that in the case of perpendicular cylinders the interaction energy for short 


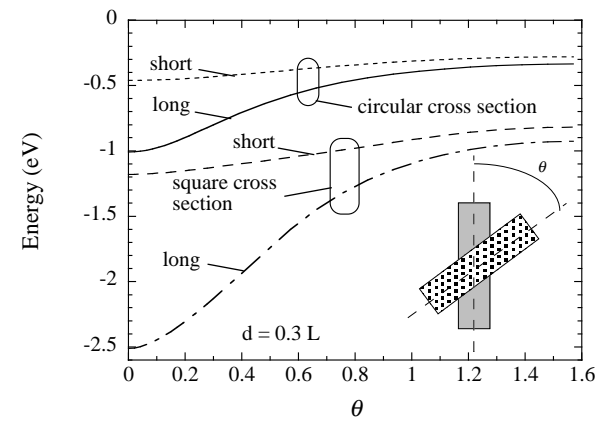

Figure 4. The rotation energy for finite gold cylinders the distance $d=0.3 L$ apart.

and long cylinders are not the same, in disagreement with the prediction from the PFA model. This fact shows that the interaction between the parts of the cylinder that do not face parts of the other one has a strong contribution to the interaction energy. In spite of the fact that the interaction between the circular cylinders shows a change of behaviour from that between two spheres, $\sim d^{-1}$, when they are fully crossed to that between two parallel cylinders, $\sim d^{-3 / 2}$, when they are aligned, we observe the strongest changes of energy in the case of square cylinders; this is due to the fact that the plane surfaces have a stronger interaction than the curved surfaces. We can also see that there is no perfect additivity in this configuration. In the case of complete alignment $(\theta=0)$ the cylinders of double length do not show double energy.

\section{Acknowledgments}

This research was sponsored by EU within the EC-contract No:012142-NANOCASE and we thank the organizing committee of the QFEXT07 for the partial support to attend the workshop.

\section{References}

[1] Casimir H B 1948 Proc. K. Ned. Akad. Wet. 51793

[2] van Kampen N G, Nijboer B R A and Schram K 1968 Phys. Lett. A 26307

[3] Gerlach E 1971 Phys. Rev. B 4393

[4] Sernelius Bo E 2001 Surface Modes in Physics (Berlin:Wiley-VCH)

[5] Sernelius Bo E 2005 Phys. Rev. B 71235114

[6] Intravaia F and Lambrecht A 2005 Phys. Rev. Lett. 94110404

[7] Derjaguin B 1934 Kolloid Z. 69155

[8] Noguez C and Román-Velázquez C E 2004 Phys. Rev. B 70, 195412

[9] Noguez C, Román-Velázquez C E, Esquivel-Sirvent R and Villarreal C 2004 Europhys. Lett., 67, 191

[10] Bergman, D J 1978 Phys. Rep. 43377

[11] Román-Velázquez C E, Noguez C and Barrera R G 2000 Phys. Rev. B 61, 10427

[12] Fuchs R 1975 Phys. Rev. B 111732

[13] Lambrecht A, Reynaud S, 2000 Eur. Phys. J. D 8309

[14] Israelachvili J N 1992 Intermolecular and Surface Forces (London:Academic Press)

[15] Sernelius Bo E and Björk P 1998 Phys. Rev. B 576592

[16] Boström M and Sernelius Bo E 2000 Phys. Rev. B 612204 